\title{
El rol moderador de la procrastinación sobre la relación entre el estrés académico y bienestar psicológico en estudiantes de pregrado
}

\section{The moderator role of procrastination in the relationship between academic stress and psychological well-being in undergraduate students}

\author{
Ariana L. Delgado-Tenorio \\ Universidad de Lima, Lima, Perú \\ ORCID: https://orcid.org/0000-0002-0831-4499 \\ Nayara A. Oyanguren-Casas* \\ Universidad de Lima, Lima, Perú \\ ORCID: https://orcid.org/0000-0002-1211-203X \\ Ariana A.I. Reyes-González \\ Universidad de Lima, Lima, Perú \\ ORCID: https://orcid.org/0000-0002-3705-5131 \\ Ángel Ch. Zegarra \\ Universidad de Lima, Lima, Perú \\ ORCID: https://orcid.org/0000-0001-5873-745X \\ Manuel E. Cueva \\ Universidad de Lima, Lima, Perú \\ ORCID: https://orcid.org/0000-0002-0402-8583
}

*Correspondencia:

Email: nayaraoyangurenc@gmail.com

\section{Citar como:}

Delgado-Tenorio, A., Oyanguren-Casas, N., ReyesGonzález, A., Zegarra, A., \& Cueva, M. (2021). El rol moderador de la procrastinación sobre la relación entre el estrés académico y bienestar psicológico en estudiantes de pregrado. Propósitos y Representaciones, 9(3), e1372. http://dx.doi.org/10.20511/pyr2021.v9n3.1372 


\section{Resumen}

El presente estudio tiene como objetivo analizar la asociación que existe entre estrés académico y bienestar psicológico, y el rol que ejerce la procrastinación académica en esta relación, en una muestra de estudiantes universitarios de pregrado. Participaron 391 estudiantes universitarios de pregrado de diversas universidades de Lima Metropolitana - Perú. Los constructos fueron medidos a partir de la adaptación española de las Escalas de Bienestar Psicológico de Ryff (SPWB), el Inventario Sistémico Cognoscitivista (SISCO SV-21) y la Escala de Procrastinación Académica (EPA). Los resultados indican que la procrastinación y el estrés académico presentan una relación negativa con respecto al bienestar psicológico. Además, la presencia de estresores y síntomas asociados al estrés académico, y la procrastinación resultan ser predictores relevantes del bienestar psicológico; sin embargo, la interacción entre ambas variables no implica un efecto significativo. Por lo tanto, se concluye que no existe un efecto moderador de la procrastinación académica en la relación entre estrés académico y bienestar psicológico. En su lugar, se halló que la procrastinación ejerce un rol moderador en la relación entre la presencia de estresores y el desarrollo de síntomas asociados al estrés académico.

Palabras clave: Procrastinación académica; Estrés académico; Bienestar psicológico; Estudiantes universitarios.

\section{Summary}

The present study aims to analyze the association between academic stress and psychological wellbeing, and the role of academic procrastination in this relationship, in a sample of undergraduate students. The sample was composed by 391 undergraduate students from various universities in Lima Metropolitana - Peru. The Spanish adaptation of Ryff's Psychological Well-being Scales (SPWB), The Cognitive Systemic Inventory (SISCO SV-21) and the Academic Procrastination Scale (EPA). Results show that procrastination and academic stress are negatively related to psychological well being. Moreover, presence of stressors and symptoms associated with academic stress, and procrastination are relevant predictors of psychological well-being; however, the interaction between both variables did not represent a significant effect; therefore, it is concluded that there is no moderating effect of academic procrastination in the relationship between academic stress and psychological well-being. Instead, procrastination was found to play a moderating role in the relationship between stressors and the development of symptoms associated with academic stress.

Keywords: Academic procrastination; Academic stress; Psychological well-being; Undergraduate students.

\section{Introducción}

En los últimos años, entre el 30\% y $60 \%$ de estudiantes de pregrado a nivel mundial procrastinan, es decir, demoran en iniciar o finalizar trabajos y tareas semanales; así como, en estudiar para exámenes o lecciones (Sultan \& Gulfisha, 2018; Mortazavi, 2016). Mientras que, en el Perú, el $60 \%$ de los estudiantes presenta un nivel medio y el $34 \%$ un nivel alto de procrastinación académica (Nava, 2018). Este comportamiento trae consecuencias como: no poder manejar adecuadamente los estudios, involucrarse en prácticas inmorales y desertar de la carrera (Hussain \& Sultan, 2010). De esta manera, la procrastinación interfiere en la voluntad de realizar las tareas y en el compromiso que tienen con las mismas (Tice \& Baumeister, 1997).

Por otro lado, una de las situaciones más complicadas que atraviesan los universitarios de pregrado, es el estrés ocasionado por las actividades académicas (Nieves et al., 2014). Este se puede manifestar mediante fatiga, tensión, mareos, insomnio, taquicardia, síntomas gastrointestinales e irritabilidad (Cecchini \& Friedman, 1987). Los estresores más resaltantes son las evaluaciones o 
exámenes, y la sobrecarga de trabajos (Jerez-Mendoza \& Oyarzo-Barría, 2015); además, están los altos niveles de competitividad y exigencia implantada por los docentes (Looker \& Gregson, 1998), los sentimientos de soledad, metas y presión familiar (Bandura, 2002; Misra \& Castillo, 2004; Watson \& Watson, 2016). De esta manera, el 30.5\% de los universitarios de pregrado estresados mostraron un descenso en sus calificaciones, no culminaron los trabajos académicos, o desertaron del curso; generando que el $86.8 \%$ se sintiera abrumado y el $61.9 \%$ sin esperanzas (Danitz \& Orsillo, 2014). En otras palabras, su bienestar psicológico se vio influenciado por el nivel de estrés académico que poseían. Por ello, un estudiante con bajo nivel de bienestar psicológico probablemente ha tenido un desequilibrio entre sus expectativas, aspiraciones y metas; considerando que su satisfacción y habilidades personales no le permitieron enfrentar situaciones cotidianas para adaptarse (Molina \& Meléndez, 2006). Esto evidencia que el bienestar psicológico es considerado como un continuo que cambia según el desarrollo y los acontecimientos de la vida del estudiante universitario (Rosa-Rodríguez, Negrón, Maldonado, Quiñones \& Toledo, 2015).

En la teoría de bienestar de Carol Ryff, el bienestar psicológico es un componente de la salud mental y crecimiento personal que representa la convicción y el sentido de que la vida es significativa (Ryff \& Keyes, 1995). Además, se relaciona con la experiencia y apreciación del sentido de libertad, responsabilidad, una percepción positiva de la vida; y se vincula con la realización de metas personales, aceptación de las adversidades, satisfacción con la vida y realización propia (Ryff \& Keyes, 1995). Es así como, Ryff (1989) plantea seis dimensiones que caracterizan al constructo de bienestar psicológico. Autoaceptación hace referencia a las evaluaciones positivas que realizamos de nosotros y de nuestra vida pasada (Ryff \& Keyes, 1995). Relaciones positivas con los otros es la percepción de la calidad de las relaciones con las personas (Ryff \& Keyes, 1995); también, se entiende como la empatía y confianza con los demás (Mayordomo, Sales, Satorres \& Meléndez, 2016). Autonomía es el sentido de la autodeterminación (Ryff \& Keyes, 1995) o capacidad de poder tomar decisiones independiente e individualmente sin que interfiera la presión social (Mayordomo et al., 2016). Dominio del ambiente consiste en la capacidad para poder manejar la vida de uno y todo lo que nos rodea (Ryff \& Keyes, 1995); además, es la habilidad de elegir o crear ambientes adecuados según nuestras condiciones psicológicas (Ryff \& Singer, 1996). Propósito de vida es la creencia de que cada individuo tiene una finalidad (Ryff $\&$ Keyes, 1995). Crecimiento personal es el desarrollo continuo (Ryff \& Keyes, 1995), enfocándose en la autorrealización y el logro del potencial personal (Ryff, 2017).

El estrés académico es aquel "proceso sistémico, de carácter adaptativo y esencialmente psicológico, el cual se presenta de manera descriptiva en tres momentos" (Barraza, 2006, p. 126). El primer momento implica una serie de inputs, demandas académicas generadoras de estrés. El segundo es la situación estresante, cuando los estresores causan un desequilibrio sistémico, manifestándose mediante síntomas. El tercero se caracteriza por realizar acciones de afrontamiento output para restaurar el equilibrio sistémico (Barraza, 2006). El Modelo Sistémico Cognoscitivista del Estrés Académico tiene cuatro hipótesis (Berrío \& Mazo, 2011). La primera involucra los componentes sistémicos-procesuales del estrés académico que implica un proceso relacional sistema-entorno (Barraza \& Silerio, 2007). La segunda establece que el estrés académico representa un estado psicológico, presentándose ante los estresores, que son "estímulos o situaciones amenazantes que desencadenan en el sujeto una reacción generalizada e inespecífica" (Barraza, 2005, p. 17). Estos estresores son: "competitividad grupal, sobrecarga de tareas, exceso de responsabilidad, interrupciones del trabajo, ambiente físico desagradable, falta de incentivos, tiempo limitado para hacer el trabajo, problemas o conflictos con los asesores y/o compañeros, las evaluaciones y el tipo de trabajo" (Barraza, 2008, p. 273). La tercera implica indicadores del desequilibrio sistémico, los cuales Barraza (2008) considera como distrés o estrés negativo que se manifiesta a través de síntomas o indicadores. Según Rossi (2001), Berrío \& Mazo (2011) los síntomas pueden ser físicos, como cefaleas, insomnio, problemas de digestión, fatiga, temblores musculares o sudoración excesiva; psicológicos, relacionados con las funciones cognitivas o emocionales tales como desconcentración, bloqueos mentales, problemas de memoria, inquietudes, ansiedad, depresión, tristeza o angustia; o comportamentales, como el ausentismo a las clases, 
desgano, conflictos, aislamiento, entre otros. Finalmente, la cuarta es la hipótesis del afrontamiento como restaurador del equilibrio sistémico, ello a partir de las estrategias de afrontamiento (Barraza \& Silerio, 2007).

La procrastinación académica es la tendencia a postergar actividades o tareas para fechas futuras, ya que el estudiante suele distraerse con otras actividades (Ellis \& Knaus, 1977). Este concepto es analizado a través de la teoría cognitivo-conductual al estar relacionado con la autorregulación académica, es decir, la capacidad para tener o mantener pensamientos, comportamientos y sentimientos que lleven a la meta (Ellis \& Knaus, 1977). Asimismo, existen características del estudiante procrastinador en tres niveles: en el cognitivo, el estudiante emplea la razón para aplazar las actividades que debe hacer, teniendo pensamientos desastrosos y rumiadores, un autoconcepto negativo y no auto eficaz (Tuckman, 2010; Guzmán 2013; Steel, 2011). A nivel conductual, se presenta la conducta impulsiva, es decir, distraerse con facilidad, incoherencia entre lo que dice que quiere hacer y lo que hace, y dificultad para tomar decisiones con rapidez (Steel, 2011); además, la poca tolerancia a la frustración o necesidad de realizar actividades placenteras con el fin de disminuir la ansiedad que le genera aplazar las tareas, la culpa y la falta de autorregulación (Guzmán, 2013). A nivel emocional, el estudiante tiene un gran miedo al fracaso y al éxito, a no obtener los resultados deseados, a tener sentimientos de culpa, ansiedad y estrés (Guzmán, 2013)

Con respecto a la relación entre estos constructos, procrastinar se relaciona con la presencia de mayores niveles de estrés académico (Sirois \& Trosti, 2012). Flett, et al (1995) afirman que dicha relación involucra una carencia en la capacidad para lidiar con estímulos adversos, además de una baja percepción de autocontrol y autoeficacia. De la misma manera, un mayor grado de estrés académico implica un deterioro en la calidad de vida y bienestar psicológico (Yataco, 2019). Es por ello que se consideró relevante abordar el estudio de la relación entre los tres constructos. En principio, el impacto adverso del estrés sobre el bienestar psicológico de estudiantes ha sido ampliamente estudiado en la literatura (e.g., Alharbi \& Smith, 2018; Adom et al., 2020; Clabaugh et al., 2021; Ribeiro et al., 2018). En la presente investigación se postula ampliar este marco al proponer un modelo de moderación en donde se introduce la procrastinación como una variable moderadora que puede incrementar la fuerza de la relación entre ambas variables. La razón en la cual se sustenta la incorporación de procrastinación es porque en las investigaciones se ha encontrado que las conductas asociadas a procrastinación como el aplazamiento de tareas y responsabilidades incrementan los niveles de estrés, lo cual tiene como consecuencia problemas de salud, dificultades emocionales, sentimientos negativos como la culpa, ansiedad, depresión y demás afecciones que incrementan el impacto negativo sobre el bienestar integral de la persona y su satisfacción con la vida (Gómez-Romero et al., 2020; Beutel et al., 2016; Wright et al., 2017; Glick et al., 2014; Pychyl et al., 2000). De este modo, evitar la realización de trabajos académicos o mostrar tendencias de procrastinación perjudica la sensación de eficacia del individuo, desencadenando factores de estrés y teniendo como efecto una mayor experiencia de estado de ánimo negativo (Gómez-Romero et al., 2020). En este sentido, el presente artículo de investigación tiene como objetivo general analizar la relación que existe entre estrés académico, bienestar psicológico y el rol que ejerce la procrastinación académica en esta interacción, específicamente, en una muestra de estudiantes universitarios de pregrado.

\section{Método}

\section{Criterios de inclusión y exclusión}

El estudio contempló como criterios de inclusión a estudiantes universitarios que residen Lima Metropolitana y se encontraran estudiando durante el periodo del segundo semestre de 2020. Como criterios de exclusión se delimitó retirar a estudiantes de posgrado, institutos, u otros centros de estudios no universitarios; además, se procuró no incluir a algún estudiante que no haya experimentado estrés durante el segundo semestre de 2020. 


\section{Participantes y proceso de muestreo}

El proceso de muestreo se desarrolló sobre un diseño no probabilístico e intencional con el objetivo de identificar casos que cumplan con los criterios de inclusión delimitados (Coolican, 2019). Un total de 391 estudiantes de universidades fueron reclutados. Entre las principales características sociodemográficas, las edades de los participantes oscilaron entre 17 y 33 años $(M=19.3, S D=$ $1.8), 66 \%$ fueron mujeres y $34 \%$ varones, en su mayoría pertenece a la misma universidad privada $(96 \%)$.

\section{Procedimiento de recolección de datos}

Una encuesta en línea fue diseñada para contener la notificación de consentimiento informado, un breve formulario de características sociodemográficas y los tres principales instrumentos de medición del estudio. Específicamente, el consentimiento informado denotaba el carácter voluntario de la participación en el estudio, el estatus de confidencialidad, la libertad de retirarse en cualquier etapa del estudio, e información de contacto de los autores. El proceso de recolección se desarrolló entre julio y octubre de 2020. El proyecto de investigación fue aprobado por el Comité de Ética e Investigación de la Facultad de Psicología de la Universidad de Lima.

\section{Medidas}

Bienestar psicológico. Carol Ryff (1989) diseñó las Escalas de Bienestar Psicológico (SPWB, por sus siglas en inglés) como un instrumento de medición en que el bienestar psicológico es operacionalizado como un constructo multidimensional compuesto por autoaceptación, relaciones positivas con otras personas, autonomía, dominio del entorno, propósito en la vida y crecimiento personal. En el presente estudio se empleó la adaptación española propuesta por Díaz et al. (2006), una versión de 29 ítems tipo Likert con seis alternativas de respuesta que indican el grado de acuerdo del participante con el enunciado presentado, que va desde Totalmente de acuerdo hasta Totalmente en desacuerdo. Las propiedades psicométricas fueron estudiadas en una muestra de 467 adultos y se reportaron niveles adecuados de confiabilidad de las puntuaciones en las seis dimensiones, tras estimar el Coeficiente Alfa $\alpha=.70-.84$. Además, los autores encontraron un buen ajuste de los datos a un modelo jerárquico con seis factores de primer orden y uno de segundo orden $\chi^{2}(345)=615.76, p<.001, C F I=.95$, RMSEA $=.04$, SRMR $=.05$.

Estrés académico. El Inventario Sistémico Cognoscitivista (SISCO) propuesto inicialmente por Barraza (2007) fue empleado para la medición del estrés académico. Este instrumento fue diseñado sobre la base de un estado del arte acerca del constructo, desarrollado por el propio autor. Tras severas revisiones, en la versión SISCO SV-21, Barraza-Macías (2018) postula las dimensiones de estresores, síntomas y estrategias de afrontamiento como necesarias para el estudio sistémico del constructo. Sin embargo, en este estudio solo se emplearon las dimensiones de estresores y síntomas, pues corresponden a los objetivos planteados. Cada una de estas dimensiones se encuentra compuesta por siete ítems tipo Likert con una escala de respuesta con seis alternativas: Nunca, Casi nunca, Rara vez, Algunas veces, Casi siempre y Siempre. Los estudios psicométricos fueron desarrollados en una muestra de 997 estudiantes entre 14 y 20 años, en que las puntuaciones en las dimensiones de estresores y síntomas demostraron niveles de confiabilidad aceptables $\alpha=.83$ y $\alpha=.87$, respectivamente. Las evidencias de validez vinculadas a la estructura interna fueron obtenidas a través de un Análisis Factorial Exploratorio que corroboró la estructura de tres dimensiones que explican el $47 \%$ de la varianza. Es importante mencionar que se optó por colapsar las categorías centrales Rara vez y Algunas veces en una sola categoría A veces para mejorar la comprensibilidad de la escala.

Procrastinación académica. Busko (1998) propuso un instrumento de medición de la procrastinación compuesto por dos subescalas; la primera se compone por 12 ítems que abordan el constructo en situaciones generales y; la segunda, por 16 ítems que cubren exclusivamente situaciones académicas. En el presente estudio se utilizó solo la Escala de Procrastinación Académica (EPA), traducida al español por Álvarez (2010). Esta versión presenta los 16 
enunciados en un formato tipo Likert con cinco alternativas de respuesta, con valores que fluctúan entre Siempre y Nunca. La adaptación fue estudiada en una muestra de 235 estudiantes de secundaria y se encontró un grado de confiabilidad de las puntuaciones apropiado $\alpha=.80$; además, se encontraron evidencias de validez vinculadas a la estructura interna a través de un análisis factorial exploratorio que respaldan la estructura unidimensional que explica el $24 \%$ de varianza.

\section{Estrategia de Análisis}

La propuesta de análisis comenzó con la exploración de las evidencias de validez y confiabilidad de las tres medidas propuestas, como sustento de su uso en el presente estudio. Específicamente, se utiliza el Análisis Factorial Confirmatorio (AFC) para evaluar el grado de ajuste de los modelos propuestos en estudios anteriores a los datos observados. En el SPWB se evaluó una estructura jerárquica con seis factores de primer orden y uno de segundo orden; en el SISCO SV-21, una estructura multidimensional de dos factores; y en el EPA se evalúa una estructura unidimensional. Dada la cantidad de alternativas de respuesta en cada escala se plantea el uso de matrices de correlaciones de Pearson como insumo para el análisis (Watkins, 2018), y el estimador de Máxima Verosimilitud Robusta (MLR) que permite abordar desviaciones con respecto a la normalidad (Wang \& Wang, 2020). El ajuste de los modelos se evalúa a partir de los indicadores Comparative Fit Index (CFI), Root Mean Squared Error of Approximation (RMSEA) y Standardized Root Mean Square Residual (SRMR). CFI $\geq .95, R M S E A \leq .05$ y $S R M R \leq .06$ indican un ajuste excelente; mientras que $C F I \geq .90, R M S E A \leq .08$ y $S R M R \leq .08$ denotan un ajuste razonable (Keith, 2019). Las cargas factoriales también fueron analizadas, esperando valores salientes $(\lambda>.3$; Howard, 2016). Los índices de modificación serán consultados con el objetivo de mejorar los ajustes planteados, considerando únicamente aquellas modificaciones que tengan un sustento conceptual (Brown, 2015). En otra instancia, la confiabilidad fue estimada a partir del Coeficiente Omega, al tener supuestos menos restrictivos que el tradicional Coeficiente Alfa (Raykov \& Marcoulides, 2017).

Tras haber demostrado las propiedades psicométricas, los puntajes factoriales derivados de los modelos de medición evaluados en CFAs serán estimados y utilizados en análisis posteriores, al representar una mejor alternativa ante los puntajes totales brutos (Mcneish \& Gordon, 2020). Un análisis exploratorio de datos será desarrollado con el objetivo de analizar la distribución y los principales estadísticos descriptivos de las medidas, así como la evaluación de los supuestos del modelo de moderación.

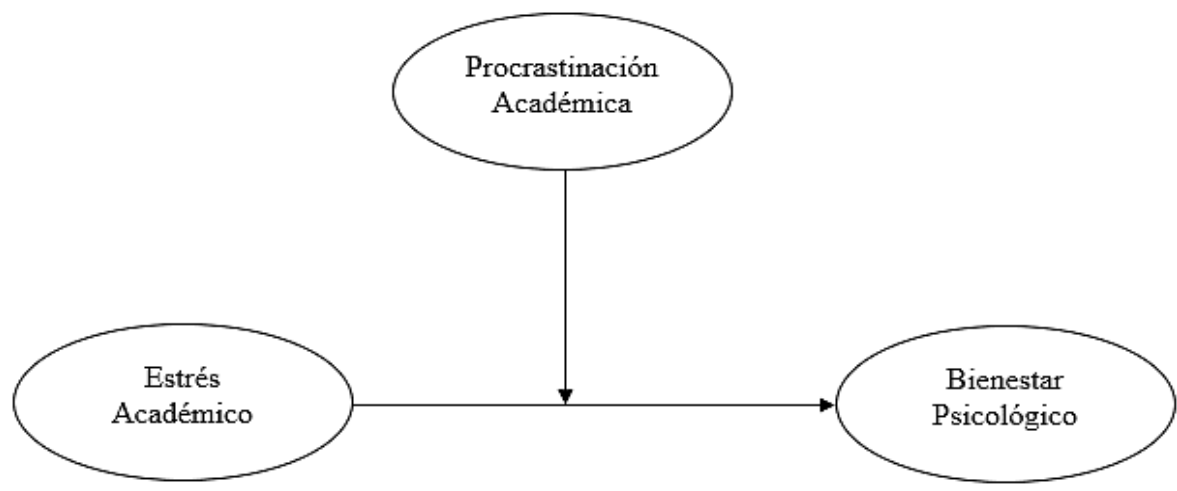

Figura 1. Procrastinación académica como moderador de la relación entre estrés académico y bienestar psicológico.

La fase final del análisis implica evaluar la pertinencia del modelo conceptual propuesto en la Figura 1 en los datos observados. En este modelo, la procrastinación académica corresponde a una variable moderadora que influye en la relación entre estrés académico y bienestar psicológico. Para ello, se plantea el uso del Factor Score Regression (FSR), con el objetivo de evaluar los modelos 
de moderación establecidos. FSR resulta en una alternativa viable ante el Modelamiento de Ecuaciones Estructurales cuando se trabaja con un modelo complejo (Devlieger, Talloen \& Rosseel, 2019). Es importante mencionar que el estudio se limita a un corte correlacional y no se pretenden establecer relaciones causa-efecto en función del modelo. Los análisis fueron desarrollados principalmente en $R$, con el paquete lavaan (Rosseel, 2012; Versión 0.6-9).

\section{Resultados}

La Tabla 1 muestra los indicadores de ajuste de los modelos de medición propuestos para las escalas SPWB, EPA y SISCO SV-21. Debido a la evidencia de un ajuste pobre de los modelos a los datos empíricos, se procedió a re-especificar las propuestas iniciales de Díaz et al. (2006), Álvarez (2010) y Barraza-Macías (2018). Siguiendo las recomendaciones de Howard (2016), el ítem 04 de la escala EPA y los ítems 13 y 14 de la escala SPWB fueron retirados del modelamiento debido a que presentaron una relación muy baja con respecto a las variables latentes que pretenden representar $(\lambda<.30)$, dicha eliminación no comprometió el grado validez, pues otros ítems abordaban un contenido similar a los retirados. Por último, acorde a recomendaciones en la literatura sobre la reespecificación de modelos considerando modificaciones ex post facto (e.g., Brown, 2015; Wang \& Wang, 2020), se procedió a liberar parámetros de correlación entre errores de aquellos ítems que emplean términos similares o cuya redacción brinda la misma idea, como en los ítems 08 y 09, e ítems 01 y 03 de la escala EPA; ítems 02 y 08, ítems 04 y 09, e ítems 08 y 22 de la escala SPWB; y, finalmente, en los ítems 12 y 13 de la escala SISCO SV-21. Como se observa en la Tabla 1, los modelos ex post facto para las tres escalas presentaron un mejor ajuste a los datos empíricos.

Tabla 1.

Evaluación del ajuste de los modelos de medición para SPWB, EPA y SISCO SV-21.

\begin{tabular}{llclllll}
\hline Escala & Modelo & $\chi^{2}(g l)$ & $\mathrm{p}$ & $\chi^{2} / g l$ & $\mathrm{CFI}$ & RMSEA (CI 90\%) & SRMR \\
\hline$S P W B$ & Díaz et al. (2006) & $924.775(371)$ & $<.001$ & 2.493 & .873 & $.067(.061, .072)$ & .062 \\
& Ex post facto & $729.383(315)$ & $<.001$ & 2.316 & .904 & $.063(.057, .069)$ & .061 \\
& & & & & & & \\
EPA & Álvarez (2010) & $596.310(104)$ & $<.001$ & 5.734 & .736 & $.119(.110, .128)$ & .074 \\
& Ex post facto & $260.487(88)$ & $<.001$ & 2.960 & .905 & $.077(.066, .088)$ & .061 \\
SISCO & Barraza-Macías & $289.288(76)$ & $<.001$ & 3.806 & .873 & $.090(.079, .101)$ & .064 \\
$S V-21$ & $(2018)$ & $164.035(75)$ & $<.001$ & 2.187 & .947 & $.058(.046, .070)$ & .050 \\
& Ex post facto & & & & & & \\
\hline
\end{tabular}

Nota. $\chi^{2}=$ Chi-cuadrado, $\mathrm{gl}=$ grados de libertad, $p=$ valor $\mathrm{p}, \mathrm{CFI}=$ Comparative Fit Index, RMSEA $=$ Root Mean Square Error of Approximation, CFI = Confidence Intervals, SRMR $=$ Standardized Root Mean Square Residual.

Los indicadores de confiabilidad indican un grado de consistencia interna apropiado, al alcanzar valores superiores al punto de corte referencial de .70 , recomendado en contextos de investigación (Kline, 2020). En primer lugar, las puntuaciones del SISCO SV-21 alcanzaron un valor de $\omega=.866$. En segundo lugar, las puntuaciones de las escalas del SPWB denotaron valores entre $\omega=.736-.862$. En tercer lugar, las puntuaciones de las escalas de síntomas y estresores del EPA indicaron $\omega=.842$ y $\omega=.795$, respectivamente.

La Tabla 2 muestra la media aritmética, desviación estándar, las puntuaciones mínimas y máximas, asimetría, curtosis y el valor p correspondiente a la prueba inferencial de normalidad de las variables procrastinación académica, bienestar psicológico juntos con sus seis dimensiones; así como, de las dimensiones estresores y síntomas del estrés académico. Estos estadísticos descriptivos se estimaron a partir de la puntuación directa de las escalas, en la perspectiva de la Teoría Clásica de los Test. 
Tabla 2.

Descriptivos de las puntuaciones totales.

\begin{tabular}{lccclll}
\hline \multicolumn{1}{l}{ Variables } & \multicolumn{1}{c}{ M } & \multicolumn{1}{c}{ DE } & \multicolumn{1}{c}{ Mín } & Máx & Asimetría & Curtosis \\
\hline Bienestar psicológico & 4.2 & 0.7 & 1.6 & 5.9 & -3.8 & 0.3 \\
Autoaceptación & 4.3 & 1.0 & 1.0 & 6.0 & -1.7 & 2.0 \\
Autonomía & 3.8 & 0.9 & 1.2 & 6.0 & -2.1 & -1.3 \\
Crecimiento & 4.8 & 0.9 & 1.3 & 6.0 & -7.1 & 3.0 \\
Dominio del entorno & 4.1 & 0.9 & 1.2 & 6.0 & -0.4 & -0.6 \\
Propósito de vida & 4.2 & 1.0 & 1.0 & 6.0 & -4.1 & -0.7 \\
Relaciones positivas & 4.3 & 1.0 & 1.0 & 6.0 & -3.2 & -1.1 \\
Procrastinación académica & 3.3 & 0.3 & 2.3 & 5.0 & 2.0 & 4.8 \\
Estresores & 3.4 & 0.7 & 1.0 & 5.0 & -1.2 & 0.8 \\
Síntomas & 6.0 & 1.3 & 2.5 & 8.8 & -1.3 & -1.0 \\
\hline
\end{tabular}

Nota M = Media, DE = Desviación Estándar, Mín = Mínimo, Máx = Máximo.

Los resultados del análisis relacional entre las variables del estudio se presentan en la Tabla 3. Específicamente, se encontraron relaciones negativas entre el Bienestar Psicológico y la Procrastinación, con tamaños del efecto moderados que oscilan entre $r=-.30$ hasta $r=-.44$. Una tendencia similar puede apreciarse al examinar las relaciones entre el Bienestar Psicológico y la escala de Estresores Académicos, pero con un tamaño del efecto pequeño, en un rango que va desde $r=-.30$ hasta $r=-.17$. Del mismo modo, se observan relaciones negativas moderadas entre las variables: Bienestar psicológico y los Síntomas del estrés relacionado a contextos académicos $(r=--33 a-.49)$. Todas las relaciones fueron estadísticamente significativas, con excepción de la relación entre Procrastinación y la presencia de Estresores Académicos $(r=.05)$.

Tabla 3.

Matriz de correlaciones entre bienestar, procrastinación y estrés académico

\begin{tabular}{lrrrrrrrrrr}
\hline Variables & 1 & 2 & 3 & 4 & 5 & 6 & 7 & 8 & 9 & 10 \\
\hline 1. Bienestar psicológico & - & & & & & & & & & \\
2. Autoaceptación & .99 & - & & & & & & & & \\
3. Autonomía & .72 & .73 & - & & & & & & & \\
4. Crecimiento personal & .92 & .91 & .65 & - & & & & & & \\
5. Dominio del entorno & .99 & .99 & .73 & .93 & - & & & & & \\
6. Propósito en la vida & .99 & .97 & .69 & .89 & .99 & - & & & & \\
7. Relaciones positivas & .75 & .73 & .52 & .72 & .76 & .72 & - & & & \\
8. Procrastinación & -.43 & -.40 & -.32 & -.37 & -.42 & -.44 & -.30 & - & & \\
9. Estresores & -.28 & -.28 & -.30 & -.25 & -.28 & -.28 & -.17 & .05 & - & \\
académicos & -.49 & -.48 & -.42 & -.42 & -.49 & -.48 & -.33 & .19 & .74 & - \\
10. Síntomas & & & & & & & \\
\hline
\end{tabular}

Nota. Matriz estimada a partir de correlaciones de Pearson. Todas las correlaciones son estadísticamente significativas, con excepción de Estresores Académicos y Procrastinación. 
En la Tabla 4 se presentan los modelos de moderación propuestos para evaluar el potencial efecto moderador de la procrastinación académica en la relación entre los síntomas del estrés académico y el bienestar psicológico, con sus respectivas dimensiones. Los resultados sugieren que tanto los síntomas como la procrastinación resultan ser predictores idóneos del bienestar psicológico, pero no existe un efecto estadísticamente significativo de la interacción entre los síntomas del estrés académico y la procrastinación. Los valores de los coeficientes estimados oscilan entre $\hat{\beta}=-.01 a \hat{\beta}=-.07$, que pueden considerarse como un tamaño del efecto sin relevancia práctica.

Tabla 4.

Modelos de moderación considerando a los síntomas del estrés académico como predictores del bienestar psicológico y procrastinación como variable moderadora.

\begin{tabular}{|c|c|c|c|c|}
\hline Criterio & Predictor & $\hat{\beta}$ & $p$ & $R^{2}$ \\
\hline Bienestar & Intercepto & .01 & .840 & $35.2 \%$ \\
\hline \multirow[t]{3}{*}{ Psicológico } & Síntomas & -.44 & $<.001$ & \\
\hline & Procrastinación & -.34 & $<.001$ & \\
\hline & Interacción & -.04 & .253 & \\
\hline \multirow[t]{4}{*}{ Autoaceptación } & Intercepto & .01 & .809 & $33.6 \%$ \\
\hline & Síntomas & -.44 & $<.001$ & \\
\hline & Procrastinación & -.31 & $<.001$ & \\
\hline & Interacción & -.05 & .172 & \\
\hline \multirow[t]{4}{*}{ Autonomía } & Intercepto & -.00 & .937 & $21.1 \%$ \\
\hline & Síntomas & -.36 & $<.001$ & \\
\hline & Procrastinación & -.25 & $<.001$ & \\
\hline & Interacción & .02 & .653 & \\
\hline \multirow{4}{*}{$\begin{array}{l}\text { Crecimiento } \\
\text { personal }\end{array}$} & Intercepto & .01 & .775 & $27.1 \%$ \\
\hline & Síntomas & -.38 & $<.001$ & \\
\hline & Procrastinación & -.29 & $<.001$ & \\
\hline & Interacción & -.07 & .107 & \\
\hline \multirow{4}{*}{$\begin{array}{l}\text { Dominio del } \\
\text { entorno }\end{array}$} & Intercepto & .01 & .833 & $35.0 \%$ \\
\hline & Síntomas & -.43 & $<.001$ & \\
\hline & Procrastinación & -.33 & $<.001$ & \\
\hline & Interacción & -.05 & .234 & \\
\hline \multirow[t]{4}{*}{ Propósito de vida } & Intercepto & .01 & .862 & $35.7 \%$ \\
\hline & Síntomas & -.42 & $<.001$ & \\
\hline & Procrastinación & -.35 & $<.001$ & \\
\hline & Interacción & -.04 & .327 & \\
\hline \multirow{4}{*}{$\begin{array}{l}\text { Relaciones } \\
\text { positivas }\end{array}$} & Intercepto & .00 & .975 & $17.0 \%$ \\
\hline & Síntomas & -.29 & $<.001$ & \\
\hline & Procrastinación & -.25 & $<.001$ & \\
\hline & Interacción & -.01 & .858 & \\
\hline
\end{tabular}

Nota. $\hat{\beta}=$ Coeficiente de correlación, $p=$ valor $\mathrm{p}, R^{2}=$ Coeficiente de determinación .

En la Tabla 5 se presentan los modelos de moderación propuestos para analizar el efecto que ejerce la procrastinación sobre la relación entre los estresores académicos y el bienestar psicológico, con sus respectivas dimensiones. Los resultados indican que la presencia de estresores 
y la procrastinación son predictores del bienestar psicológico, pero su interacción no denota un efecto estadísticamente significativo. Los valores de los coeficientes estimados oscilan entre $\hat{\beta}=$ $-.03 a \hat{\beta}=-.05$, que pueden considerarse como un tamaño del efecto sin relevancia práctica.

\section{Tabla 5.}

Modelos de moderación considerando a los estresores académicos como predictores del bienestar y procrastinación como variable moderadora.

\begin{tabular}{|c|c|c|c|c|}
\hline Criterio & Predictor & $\hat{\beta}$ & $p$ & $R^{2}$ \\
\hline \multirow[t]{4}{*}{ Bienestar Psicológico } & Intercepto & .00 & .970 & $24.5 \%$ \\
\hline & Estresores & -.27 & $<.001$ & \\
\hline & Procrastinación & -.41 & $<.001$ & \\
\hline & Interacción & -.03 & .471 & \\
\hline \multirow[t]{4}{*}{ Autoaceptación } & Intercepto & .00 & .964 & $22.7 \%$ \\
\hline & Estresores & -.26 & $<.001$ & \\
\hline & Procrastinación & -.38 & $<.001$ & \\
\hline & Interacción & -.04 & .388 & \\
\hline \multirow[t]{4}{*}{ Autonomía } & Intercepto & -.00 & .955 & $18.2 \%$ \\
\hline & Estresores & -.28 & $<.001$ & \\
\hline & Procrastinación & -.31 & $<.001$ & \\
\hline & Interacción & .05 & .285 & \\
\hline \multirow[t]{4}{*}{ Crecimiento personal } & Intercepto & .00 & .953 & $19.4 \%$ \\
\hline & Estresores & -.24 & $<.001$ & \\
\hline & Procrastinación & -.35 & $<.001$ & \\
\hline & Interacción & -.05 & .260 & \\
\hline \multirow[t]{4}{*}{ Dominio del entorno } & Intercepto & .00 & .969 & $24.6 \%$ \\
\hline & Estresores & -.27 & $<.001$ & \\
\hline & Procrastinación & -.40 & $<.001$ & \\
\hline & Interacción & -.03 & .455 & \\
\hline \multirow[t]{4}{*}{ Propósito de vida } & Intercepto & .00 & .969 & $26.0 \%$ \\
\hline & Estresores & -.26 & $<.001$ & \\
\hline & Procrastinación & -.42 & $<.001$ & \\
\hline & Interacción & -.03 & .452 & \\
\hline \multirow[t]{4}{*}{ Relaciones positivas } & Intercepto & -.00 & .970 & $11.6 \%$ \\
\hline & Estresores & -.15 & .002 & \\
\hline & Procrastinación & -.30 & $<.001$ & \\
\hline & Interacción & .04 & .467 & \\
\hline
\end{tabular}

Nota. $\hat{\beta}=$ Coeficiente de correlación, $p=$ valor $\mathrm{p}, R^{2}=$ Coeficiente de determinación .

La Figura 2 presenta gráficamente los dos principales modelos de moderación planteados y sus parámetros estimados, considerando la medida global de bienestar psicológico como variable dependiente. Como se mencionó en los párrafos anteriores, no se encontró un efecto relevante en la interacción entre procrastinación y las dos dimensiones del estrés académico evaluadas, pero ambas variables por separado resultan en predictores estadísticamente significativos del bienestar psicológico. 

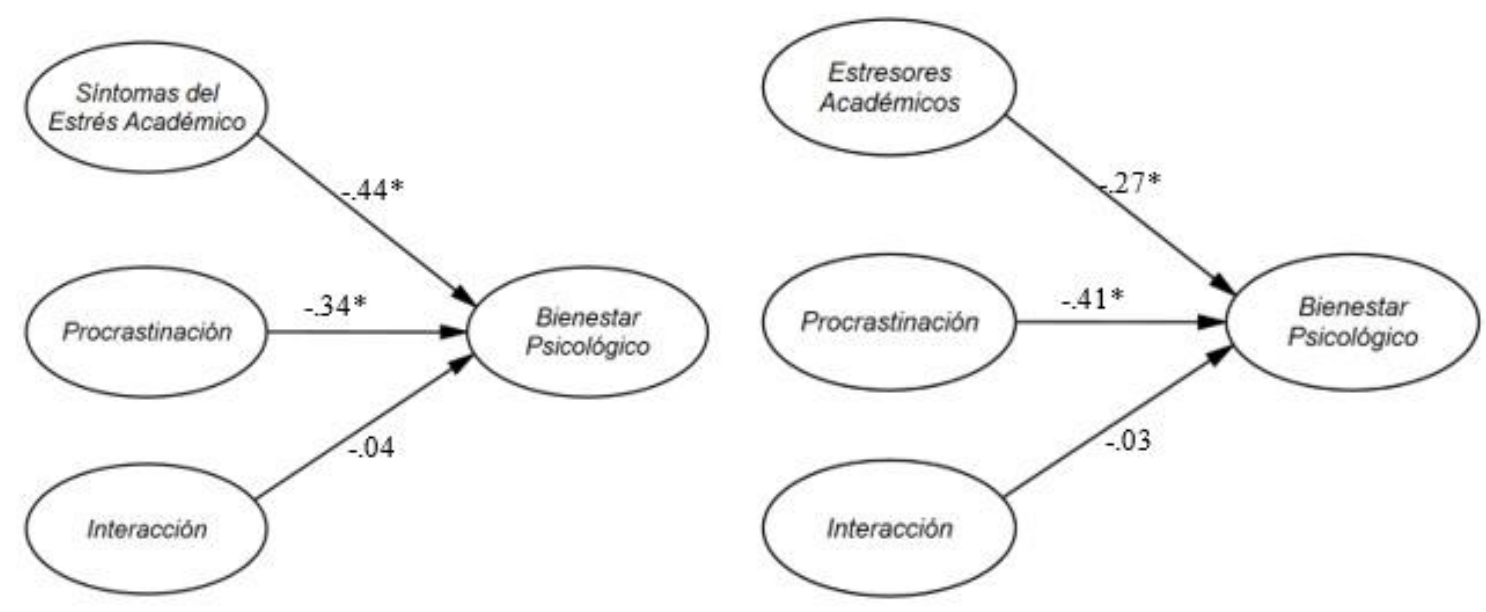

Figura 2. Representación gráfica de los principales modelos de moderación

Finalmente, para ampliar el estudio de la relación entre estas variables se evaluó la pertinencia de un tercer modelo de moderación en donde la variable dependiente son los síntomas del estrés académico y la independiente corresponde a la presencia de estresores. En la Tabla 6 se evidencia que la interacción entre la procrastinación y la presencia de estresores actúan como un predictor estadísticamente significativo para el desarrollo de síntomas del estrés académico. Esto implica evidencias a favor de que la procrastinación ejerce un rol moderador en la relación entre estas variables.

Tabla 6.

Modelo de Moderación Considerando a los Estresores Como Predictores de los Síntomas del Estrés Académico y Procrastinación Como Variable Moderadora.

\begin{tabular}{llllc}
\hline Criterio & Predictor & $\hat{\beta}$ & $p$ & $R^{2}$ \\
\hline Síntomas del & Intercepto & .00 & .910 & $56.8 \%$ \\
Estrés & Estresores & .72 & $<.001$ & \\
& Procrastinación & .16 & $<.001$ & \\
& Interacción & -.08 & .031 & \\
\hline
\end{tabular}

Nota. $\hat{\beta}=$ Coeficiente de correlación, $p=$ valor $\mathrm{p}, R^{2}=$ Coeficiente de determinación.

\section{Discusión}

En el estudio sobre la relación entre procrastinación y estrés académico se brindan evidencias que sugieren que, individuos que tienden a procrastinar más en tareas vinculadas a entornos académicos, suelen experimentar mayores niveles de estrés académico (Sirois \& Trosti, 2012). Los mecanismos por los que ocurre dicha relación involucran una carencia en la capacidad para lidiar con estímulos adversos, pues aquellas personas que incurren en actos de procrastinación presentan una baja percepción de autocontrol y autoeficacia (Flett et al, 1995). En otra instancia, los estudiantes que presentan niveles altos de estrés académico tienen una menor calidad de vida; así como un grado de bienestar psicológico deteriorado; debido a que, se perciben como ineficaces al resolver problemas académicos (Yataco, 2019). Por estos motivos, se consideró importante abordar el estudio de la relación entre estos tres constructos. En un inicio, se postuló que la procrastinación podría ejercer un rol moderador en la relación entre estrés académico y bienestar psicológico, pues investigaciones sugieren que la procrastinación podría modificar los niveles de estrés dependiendo de si se utiliza como una estrategia activa o maladaptativa (Sirois \& Kitner, 2015). 
Los resultados obtenidos indican que, tanto la procrastinación como el estrés académico, ya sea la simple presencia de estresores o el desarrollo de síntomas, presentan una relación negativa entre pequeña a moderada con respecto al bienestar psicológico, este hallazgo es congruente con otros estudios en la literatura que abordan la relación entre estos constructos (e.g., Hernández, 2020). De esta manera, ambas variables tienen una capacidad predictiva importante para la predicción de los niveles de bienestar psicológico, en sus diversas dimensiones. No obstante, se encontró que la procrastinación académica no ejerce un rol moderador en la relación entre el estrés académico y el bienestar psicológico en estudiantes universitarios de pregrado de Lima Metropolitana. En otras palabras, la procrastinación académica no cumple un rol moderador entre ambas variables; por lo tanto, se rechaza la hipótesis planteada en la presente investigación.

En un análisis secundario, se encontró que la procrastinación académica cumple un rol moderador entre la presencia de estresores y el desarrollo de síntomas asociados al estrés académico. En coherencia con estos hallazgos, Sirois (2004) evidenció que, aquellos estudiantes que suelen procrastinar presentan mayores niveles de estrés comparado con los no procrastinadores, recalcando además que, la procrastinación aumenta la intensidad del estrés. Mencionado ello, a mayores niveles de estrés académico, puede existir mayor preponderancia a aplazar las actividades académicas y mayores razones para hacerlo, y viceversa. Adicionalmente, en el estudio realizado por Córdova (2018), se encontró que existe una relación altamente significativa y positiva entre los estresores como las exigencias y demandas académicas junto con la procrastinación académica porque los estudiantes universitarios escogen realizar actividades extracurriculares que pueden compartir con sus amigos, evadiendo así las actividades académicas y experimentando satisfacción al hacerlo (Sobalvarro, 2008). Asimismo, en cuanto a la dimensión síntomas y la procrastinación académica también se evidencia una relación altamente significativa y positiva (Córdova, 2018), donde se evidencia la presencia de altos niveles de estrés académico puesto que la sintomatología del estrés como irritabilidad, distracción, entre otros, se manifiesta cuando las actividades académicas son consideradas complicadas, poco atractivas y que conllevan esfuerzo (Neidhardt, Weinstein \& Conry, 1989; Ferrari, Mason \& Hammer, 2006). Todo ello demuestra que, mientras más frecuentemente los estudiantes universitarios perciban estímulos estresores y tengan reacciones comportamentales, psicológicas y físicas, optarán por postergar sus actividades académicas (Hidalgo, 2019).

En lo que concierne a la correlación entre bienestar psicológico y procrastinación académica, se determinó que esta es estadísticamente significativa, negativa y moderada. Debido a que, aplazar actividades académicas tiene un efecto negativo en el rendimiento académico (Ackerman \& Gross, 2005; Balkis, 2013), lo cual tendrá un impacto negativo en el bienestar psicológico de los estudiantes procrastinadores; pues, cuando se presentan dificultades al momento de realizar trabajos o estudiar para evaluaciones, optan por postergarlos, lo que les genera incertidumbre respecto a su autoconcepto y autoeficacia (Carranza, como se citó en Hernández, 2016). En relación con ello, Hernández (2016) encontró en su investigación que el bienestar psicológico y la procrastinación académica tienen una correlación negativa y alta; con predominio de ciertas dimensiones como el crecimiento personal, autoaceptación y relaciones positivas porque al ser parte importante del funcionamiento psicológico positivo incrementa la calidad de las experiencias tanto propias como con los demás, donde se desarrollan habilidades y el potencial para sentirse capaces de enfrentar cualquier reto académico (Ryff, 1989; Ryff \& Keyes, 1995; Hernández, 2016). En cuanto a las dimensiones propósito de vida y autonomía, se encontró una correlación negativa y baja, debido a que procrastinar refleja poco la independencia del estudiante para tomar sus decisiones y dejar que estas influyan en su vida (Ryff \& Keyes, 1995). Finalmente, con respecto a la correlación entre bienestar psicológico y estrés académico, considerando las dimensiones de estresores y síntomas, se encontró que estas son negativas y estadísticamente significativas, con un tamaño del efecto pequeño y moderado, respectivamente. Dichos resultados son congruentes con el estudio realizado por Hernández (2020), donde encontró que el bienestar psicológico y el estrés académico junto con sus estresores y síntomas mantienen una relación altamente significativa, negativa y moderada, manifestando así la importancia y el empeño que 
otorgan los estudiantes a sus estudios. En relación con las dimensiones del bienestar psicológico, las que más resaltan son dominio del entorno y relaciones positivas, debido a que implica asumir responsabilidades y confiar en quienes rodean al estudiante universitario para tener una mejor adaptación al medio académico (Selye, 1976). Ello es reforzado por Lara, Saldaña, Fernández \& Delgadillo (2015) quienes indican que aquellos estudiantes que perciben su entorno académico como grato presentan mejor salud y calidad de vida.

En síntesis, tanto la procrastinación como el estrés académico, ya sea la simple presencia de estresores o el desarrollo de síntomas, presentan una relación negativa entre pequeña a moderada respecto al bienestar psicológico. Sin embargo, se halló que la procrastinación académica no cumple un rol moderador en la relación entre estrés académico sobre el bienestar psicológico. Adicionalmente, se evidenció que la procrastinación académica cumple un rol moderador entre la presencia de estresores y el desarrollo de síntomas asociados al estrés académico. Así mismo, se determinó una correlación estadísticamente significativa, negativa y moderada entre el bienestar psicológico y la procrastinación académica; así como, una correlación estadísticamente significativa, negativa y de pequeña a moderada entre el bienestar psicológico y estrés académico, considerando las dimensiones de estresores y síntomas.

Es importante reconocer que el estudio presenta como limitación la imposibilidad de realizar inferencias de los resultados obtenidos sobre una población particular debido a la naturaleza del proceso de muestreo desarrollado y a la relativa homogeneidad presente en la muestra alcanzada. Además, las características de los cuestionarios de autoreporte comprometen la validez de los resultados en función al grado de honestidad que los participantes demostraron al responder los ítems. Por estos motivos, se recomienda que futuros estudios contrasten las relaciones encontradas en muestras con características sociodemográficas más diversas, con el objetivo de ampliar la validez externa del estudio.

\section{Referencias}

Ackerman, D., \& Gross, B. (2005). My instructor made me do it: Task characteristics of procrastination [Mi instructor me hizo hacerlo: características de la tarea de la procrastinación]. Journal of Marketing Education, 27(1), 5-13. https://www.researchgate.net/publication/247753031_My_Instructor_Made_Me_Do_It_T ask_Characteristics_of_Procrastination

Adom, D., Chukwuere, J. \& Osei, M. (2020). Academic Stress among Faculty and Students in Higher Institution [Estrés académico entre profesores y estudiantes de instituciones superiores]. Pertanika Journal of Social Sciences \& Humanities, 28(2), 1055-1064. https://www.researchgate.net/publication/342410787_Review_Academic_Stress_among_ Faculty_and_Students_in_Higher_Institutions

Alharbi, E. \& Smith, A. (2018). Review of the Literature on Stress and Wellbeing of International Students in English-Speaking Countries [Revisión de la literatura sobre estrés y bienestar internacional Estudiantes en países de habla inglesa]. International Education Studies, 11(6), 22-44. https://files.eric.ed.gov/fulltext/EJ1180852.pdf

Álvarez, O. (2010). Procrastinación general y académica en una muestra de estudiantes de secundaria de Lima metropolitana. Persona, 13, 159-177. https://www.redalyc.org/pdf/1471/147118212009.pdf

Balkis, M. (2013). Academic procrastination, academic life satisfaction and academic achievement: The mediation role of rational beliefs about studying [Dilación académica, satisfacción con la vida académica y rendimiento académico: el papel de mediación de las creencias racionales sobre el estudio]. Journal of Cognitive and Behavioral Psychotherapies, 13(1), 57-74. https://psycnet.apa.org/record/2013-13923-004

Bandura, A. (2002). Social cognitive theory in cultural context [Teoría cognitiva social en un contexto cultural]. Applied Psychology International Review, 51, 269-290. http://dx.doi.org/10.111/1464-0597.00092 
Barraza, A. (2005). Características del estrés académico de los alumnos de educación media superior. Investigación Educativa Duranguense, 4, 15-20. https://dialnet.unirioja.es/servlet/articulo?codigo $=2880918$

Barraza, A. (2006). Un modelo conceptual para el estudio del estrés académico. Revista Electrónico $\begin{array}{llll}\text { de Psicología 110-129. } & \text { Iztacala, }\end{array}$ http://www.revistas.unam.mx/index.php/repi/article/view/19028/18052

Barraza, A. (2008). El estrés académico en alumnos de maestría y sus variables moduladoras: un diseño de diferencia de grupos. Avances en Psicología Latinoamericana, 26(2), 270-289. https://www.redalyc.org/pdf/799/79926212.pdf

Barraza-Macías, A. (2018). Inventario SISCO SV-21 inventario sistémico cognoscitivista para el estudio del estrés académico. Segunda versión de 21 items [versión Adobe Digital Editions]. http://www.ecorfan.org/libros/Inventario_SISCO_SV21/Inventario_sist\%C3\%A9mico_cognoscitivista_para_el_estudio_del_estr\%C3\%A9s.pd $\mathrm{f}$

Barraza, A., \& Silerio, J. (2007). El estrés académico en alumnos de educación media superior: un estudio comparativo. Investigación Educativa Duranguense, 7, 48-65. https://dialnet.unirioja.es/servlet/articulo?codigo $=2358918$

Berrío, N., \& Mazo, R. (2011). El estrés académico. Revista de Psicología (Universidad de Antioquía), 3(2), 65-82. https://dialnet.unirioja.es/servlet/articulo?codigo=4865240

Beutel, M., Klein, E., Aufenanger, S., Brähler, E., Dreier, M., Müller, K., Quiring, O., Reinecke, L., Schmutzer, G., Stark, B. \& Wölfling, K. (2016). Procrastination, Distress and Life Satisfaction across the Age Range - A German Representative Community Study [Dilación, angustia y satisfacción con la vida en todos los rangos de edad: un estudio comunitario representativo de Alemania]. PLoS One,11(2), 1-12. https://www.ncbi.nlm.nih.gov/pmc/articles/PMC4752450/

Brown, T. A. (2015). Confirmatory factor analysis for applied research (2nd ed.). New York, NY: The Guilford Press

Busko, D. (1998). Causes and consequences of perfectionism and procrastination: A structural equation model (Tesis de licenciatura, Universidad de Guelph). https://citeseerx.ist.psu.edu/viewdoc/download?doi=10.1.1.456.4450\&rep=rep1\&type=pd $\mathrm{f}$

Cecchini, J., J. \& Friedman, N. (1987). First-year dental students: Relationship between stress and performance [Primer año de estudiantes de odontología: relación entre el estrés y el desempeño]. International Journal of Psychosomatics, 34(3), 17-19. https://psycnet.apa.org/record/1988-34146-001

Clabaugh, A., Duque, J. \& Fields, L. (2021). Academic Stress and Emotional Well-Being in United States College Students Following Onset of the COVID-19 Pandemic [Estrés académico y bienestar emocional en estudiantes universitarios de Estados Unidos luego del inicio de la pandemia COVID-19]. Frontiers in Psychology, 12, 1-8. https://www.ncbi.nlm.nih.gov/pmc/articles/PMC8010317/

Coolican, H. (2019). Research methods and statistics in psychology (7th ed). New York, NY: Routledge

Córdova, C. (2018). Procrastinación y estrés académico en estudiantes de la Universidad Nacional de Ingeniería, 2016 (Tesis de licenciatura, Universidad Peruana Unión). https://repositorio.upeu.edu.pe/bitstream/handle/20.500.12840/1298/Carlos_Tesis_Titulo 2018.pdf?sequence $=5$ \&isAllowed $=\mathrm{y}$

Danitz, S., \& Orsillo, S. (2014). The mindful way through the semester: An investigation of the effectiveness of an acceptance based behavioral therapy program on psychological wellness in first-year students [El camino consciente a lo largo del semestre: una investigación de la eficacia de un programa de terapia conductual basada en la aceptación sobre el bienestar psicológico en estudiantes de primer año]. Behaviour Modification, 38(4), 549-566. https://pubmed.ncbi.nlm.nih.gov/24452372/ 
Devlieger, I., Talloen, W., \& Rosseel, Y. (2019). New developments in factor score regression: Fit índices and a model comparison test [Nuevos desarrollos en la regresión de puntuación de factores: índices de ajuste y una prueba de comparación de modelos]. Educational and Psychological Measurement, 79(6), 1017-1037. http://dx.doi.org/10.1177/0013164419844552

Díaz, D., Rodríguez-Carvajal, R., Blanco, A., Moreno-Jiménez, B., Gallardo, I., Valle, C., \& van Dierendonck, D. (2006). Adaptación española de las escalas de bienestar psicológico de Ryff. Psicothema, 18(3), 572-577. http://www.psicothema.com/pdf/3255.pdf

Ellis, A., \& Knaus, W. J. (1977). Overcoming procrastination [Superando la procrastinación]. Institute for Rational Living.

Ferrari, J., Mason, C., \& Hammer, C. (2006). Procrastination as a predictor of Task Perceptions: examining delayed and non-delayed tasks across varied deadlines [La procrastinación como predictor de las percepciones de las tareas: examinar las tareas retrasadas y no retrasadas en distintos plazos]. Individual Differences Research, 4(1), 28-36. https://www.researchgate.net/publication/276354422_Procrastination_as_a_Predictor_of_ Task_Perceptions_Examining_Delayed_and_Nondelayed_Tasks_Across_Varied_Deadlines

Flett, G. L., Blankstein, K. R., \& Martin, T. R. (1995). Procrastination and Task Avoidance. En J. R. Ferrari, J. L. Johnson y W. G. McCown (Eds.), Procrastination, negative selfevaluation, and stress in depression and anxiety [Procrastinación, autoevaluación negativa y estrés en depresión y ansiedad] (pp. 137-167). http://dx.doi.org/10.1007/978-1-48990227-6_7

Glick, D. M., Millstein, D. J., \& Orsillo, S. M. (2014). A preliminary investigation of the role of psychological inflexibility in academic procrastination [Una investigación preliminar del papel de la inflexibilidad psicológica en la procrastinación académica]. Journal of Contextual Behavioral Science, 3(2), 81-88. https://doi.org/10.1016/j.jcbs.2014.04.002

Gómez-Romero, M., Tomás-Sábado, J., Montes-Hidalgo, J., Brando-Garrido, C., Cladellas, R., y Limonero, J. (2020). Procrastinación académica y riesgo de conducta suicida en jóvenes universitarios: el papel de la regulación emocional. Revista Ansiedad y Estrés, 26(2-3), 112-119. https://www.sciencedirect.com/science/article/abs/pii/S1134793720300269?via\%3Dihub

Guzmán, D. (2013). Procrastinación: Una mirada clínica (Tesis de maestría, Instituto Superior de Estudios Psicológicos). https://www.academia.edu/13227195/Procrastinacion_TEMA

Hernández, G. (2016). Procrastinación académica, motivos de procrastinación y bienestar psicológico en alumnos de ingeniería industrial de una universidad de Trujillo (Tesis de titulación, Universidad Privada del Norte). https://repositorio.upn.edu.pe/bitstream/handle/11537/10716/Hern\%c3\%a1ndez\%20Malc a\%20Gabriel\%20Fernando.pdf?sequence $=1 \&$ isAllowed $=\mathrm{y}$

Hernández, N. (2020). Procrastinación académica, estrés académico y bienestar psicológico en estudiantes de psicología de una universidad privada de Chicha, 2020 (Tesis de maestría, Universidad Privada de Chincha). https://repositorio.usmp.edu.pe/bitstream/handle/20.500.12727/7822/HERNANDEZ_CN. pdf? sequence $=1$ \&isAllowed $=\mathrm{y}$

Hidalgo, L. (2019). Estrés académico y procrastinación académica en estudiantes en una universidad privada de Trujillo (Tesis de licenciatura, Universidad Privada del Norte). Recuperado de https://repositorio.upn.edu.pe/handle/11537/23175

Howard, M. C. (2016). A review of exploratory factor analysis decisions and overview of current practices: What are we doing and how can we improve? [Una revisión de las decisiones de análisis factorial exploratorio y una descripción general de las prácticas actuales: ¿Qué estamos haciendo y cómo podemos mejorar?] International Journal of Human-Computer Interaction, 32(1), 51-62. doi: http://dx.doi.org/10.1080/10447318.2015.1087664

Hussain, I., \& Sultan, S. (2010). Analysis of procrastination among university students [Análisis de la procrastinación entre estudiantes universitarios]. Procedia Social and Behavioral Sciences, 5, 1897-1904. doi: http://dx.doi.org/10.1016/j.sbspro.2010.07.385 
Jerez-Mendoza, M., \& Oyarzo-Barría, C. (2015). Estrés académico en estudiantes del Departamento de Salud de la Universidad de Los Lagos Osorno. Revista chilena de neuropsiquiatría, 53(3), 149-157. Recuperado de https://scielo.conicyt.cl/pdf/rchnp/v53n3/art02.pdf

Keith, T. Z. (2019). Multiple regression and beyond. An introduction to multiple regression and structural equation modeling (3rd ed.). New York, NY: Routledge

Kline, R. B. (2020). Becoming a behavioral science researcher. A guide to producting research that matters [Convertirse en un investigador de ciencias del comportamiento. Una guía para producir investigaciones que importan] (2 ed). New York, NY: The Guilford Press.

Lara, N., Saldaña, Y., Fernández, N., \& Delgadillo, H. (2015). Salud, calidad de vida y entorno universitario en estudiantes mexicanos de una universidad pública. Hacia la Promoción de la Salud, 20(2), 102-117. Recuperado de http://www.scielo.org.co/pdf/hpsal/v20n2/v20n2a08.pdf

Looker, T., \& Gregson, O. (1998). Superar el estrés. Madrid, España: Pirámide.

Mayordomo, T., Sales, A., Satorres, E., \& Meléndez, J. (2016). Bienestar psicológico en función de la etapa de vida, el sexo y su interacción. Pensamiento Psicológico, 14(2), 101-112. Recuperado de http://www.scielo.org.co/pdf/pepsi/v14n2/v14n2a08.pdf

McNeish, D., \& Wolf, M.G. (2020). Thinking twice about sum scores [Pensando dos veces en la suma de puntajes]. Behavioral Research Methods, 52, 2287-2305. doi: http://dx.doi.org/10.3758/s13428-020-01398-0

Misra, R., \& Castillo, L. (2004). Academic stress among college students: Comparison of American and international students [Estrés académico entre estudiantes universitarios: comparación de estudiantes americanos e internacionales]. International Journal of Stress Management, 11(2), 132-148. Recuperado de https://sci-hub.se/10.1037/1072-5245.11.2.132

Molina, C.J., \& Meléndez, J. C. (2006). Bienestar psicológico en envejecientes de la República Dominicana. Geriátrika, 22(3), 97-105. Recuperado de https://www.uv.es/melendez/envejecimiento/bienestar.pdf

Mortazavi, F. (2016). The prevalence of academic procrastination and its association with medical student's well-being status [La prevalencia de la procrastinación académica y su asociación con el estado de bienestar en estudiantes de medicina]. International Journal of Humanities and Cultural Studies, 3(2), 1256-1268. Recuperado de https://www.researchgate.net/publication/311844440_The_prevalence_of_academic_proc rastination_and_its_association_with_medical_students\%27_well-being_status

Nava, J. (2018). Procrastinación en estudiantes universitarios de la Facultad de Psicología de una universidad privada del Cercado de Lima (Tesis de licenciatura, Universidad Inca Garcilaso de la Vega). Recuperado de http://repositorio.uigv.edu.pe/bitstream/handle/20.500.11818/3394/TRAB.SUF.PROF_Jo rge\%20Enrique\%20Nava\%20Ore\%20Garro.pdf?sequence=2\&isAllowed=y

Neidhardt, J., Weinstein, M., \& Conry, R. (1989). Seis programas para prevenir y controlar el estrés. Madrid, España: Deusto.

Nieves, Z., Otero, I., Mabiala, C., Malonda, H., \& Guimbi, U. (2014). Principales manifestaciones del estrés académico en estudiantes de la carrera de medicina de la Universidad "Onze de novembro" Cabinda, Angola. Revista Psicoespacios, 8(12), 402-420. Recuperado de https://webcache.googleusercontent.com/search?q=cache:R4FOgyqY2mYJ:https://dialnet .unirioja.es/descarga/articulo/4925167.pdf $+\& c d=1 \&$ hl=es\&ct=clnk\&gl=pe

Pychyl, T. A., Lee, J., Thibodeau, R., \& Blunt, A. (2000). Five days of emotions: An experience sampling study of undergraduate student procrastination [Cinco días de emociones: una experiencia que muestra el estudio de la procrastinación de los estudiantes de pregrado]. Journal of Social Behavior and Personality, 15(5), 239-254. https://psycnet.apa.org/record/2002-10572-019

Raykov, T., \& Marcoulides, G. A. (2017). Thanks Coefficient Alpha, we still need you! [iGracias Coefficient Alpha, ¡todavía te necesitamos!] Educational and Psychological Measurement, 79(1), 200-210. doi: http://dx.doi.org/10.1177/0013164417725127 
Ribeiro, Í., Pereira, R., Freire, I., de Oliveira, B., Casotti, C. \& Boery, E. (2018). Stress and Quality of Life Among University Students: A Systematic Literature Review [Estrés y calidad de vida en estudiantes universitarios: revisión sistemática de la literatura]. Health Professions Education, 4(2), 70-77. Recuperado de https://www.sciencedirect.com/science/article/pii/S2452301117300305

Rosa-Rodríguez, Y., Negrón, N., Maldonado, Y., Quiñones, A., \& Toledo, N. (2015). Dimensiones de bienestar psicológico y apoyo social percibido con relación al sexo y nivel de estudio en universitarios. Avances en Psicología Latinoamericana, 33, 31-43. Recuperado de http://www.scielo.org.co/pdf/apl/v33n1/v33n1a03.pdf

Rosseel, Y. (2012). lavaan: An R Package for Structural Equation Modeling [lavaan: un paquete R para el modelado de ecuaciones estructurales]. Journal of Statistical Software, 48(2), 1-36. Recuperado de https://www.jstatsoft.org/v48/i02/.

Rossi, R. (2001). Para superar el estrés. Barcelona, España: De Vecchi

Ryff, C. (1989). Happiness is everything, or is it? Explorations on the meaning of psychological well-being [La felicidad lo es todo, ¿o no? Exploraciones sobre el significado del bienestar psicológico]. Journal of Personality and Social Psychology, 57(6), 1069-1081. https://pdfs.semanticscholar.org/0b7c/bc0e7b5946b39778784a2167019eebd53e52.pdf?_g $\mathrm{a}=2.181014948 .223177416 .1607351385-1716770294.1600788620$

Ryff, C. (2017). Eudaimonic well-being, inequality, and health: Recent findings and future directions [Bienestar, desigualdad y salud eudemónicos: hallazgos recientes y direcciones futuras]. International Review of Economics, 64(2), 159-178. https://www.ncbi.nlm.nih.gov/pmc/articles/PMC5645055/

Ryff, C., \& Keyes, C. (1995). The Structure of Psychological Well-Being Revisited [Revisión de la estructura del bienestar psicológico]. Journal of Personality and Social Psychology, 69(4),719-727. http://www.midus.wisc.edu/findings/pdfs/830.pdf

Ryff, C., \& Singer, B. (1996). Psychological Well-Being: Meaning, Measurement, and Implications for Psychotherapy Research [El bienestar psicológico: significado, medición e implicaciones para la investigación en psicoterapia]. Psychotherapy and Psychosomatics, 65, 14-23. https://www.researchgate.net/publication/14366097_Psychological_WeilBeing_Meaning_Measurement_and_Implications_for_Psychotherapy_Research

Selye, H. (1976). Stress without distress. En G. Serban (Ed.), Psychopathology of human adaptation (pp. 137-146). New York, NY: Springer.

Sirois, F. (2004). Procrastination and intentions to perform health behaviors: The role of selfefficacy and the consideration of future consequences [Procrastinación e intenciones de realizar conductas saludables: el papel de la autoeficacia y la consideración de las consecuencias futuras]. Personality and Individual Differences, 37(1), 115-128. https://www.researchgate.net/publication/222518941_Procrastination_and_intentions_to_ perform_health_behaviors_The_role_of_self-

efficacy_and_the_consideration_of_future_consequences

Sirois, F., \& Kitner, R. (2015). Less adaptive or more maladaptive? A meta-analytic investigation of procrastination and coping [¿Menos adaptativo o más inadaptado? Una investigación metaanalítica de la procrastinación y el afrontamiento]. European Journal of Personality, 29(4), 433-444. http://dx.doi.org/10.1002/per.1985

Sirois, F., \& Tosti, N. (2012). Lost in the moment? And investigation of procrastination, mindfulness, and well-being [¿Perdido en el momento? Una investigación sobre la procrastinación, la atención plena y el bienestar]. Journal of Rational-Emotive \& Cognitive-Behavior Therapy, 30, 237-248. http://dx.doi.org/10.1007/s10942-012-0151-y

Sobalvarro, C. (2008). La toma de decisiones y la procrastinación (Estudio comparativo en ejecutivos de empresas) (Tesis de titulación, Universidad de San Carlos de Guatemala). http://www.repositorio.usac.edu.gt/14295/1/13\%20T\%201503.pdf

Steel, P. (2011). Procrastinación: Por qué dejamos para mañana lo que podemos hacer hoy. Grijalbo. 
Sultan, S., \& Gulfisha, Q. (2018). A study of academic motivation, procrastination, and stress among university students [Un estudio de la motivación académica, la procrastinación y el estrés entre los estudiantes universitarios]. International Journal of Research Culture Society, 2(7), 22-26. https://www.researchgate.net/publication/335465863_A_Study_of_Academic_motivation _procrastination_and_stress_among_university_students

Tice, D., \& Baumeister, R. (1997). Longitudinal Study of Procrastination, Performance, Stress, and Health: The Costs and Benefits of Dawdling [Estudio longitudinal de la procrastinación, el desempeño, el estrés y la salud: los costos y beneficios de perder el tiempo]. Psychological Science, $8(3)$ 454-458. https://www.researchgate.net/publication/240286119_Longitudinal_Study_of_Procrastina tion_Performance_Stress_and_Health_The_Costs_and_Benefits_of_Dawdling

Tuckman, B. (2010). Getting Over the "ABD” Hump: The secret is avoiding procrastination. En R. Calabrese y P. Smith (Eds.), The doctoral student's advisor and mentor: Sage advice from experts [El asesor y mentor del estudiante de doctorado: sabios consejos de expertos] (pp. 53-56). United.

Watson, J., \& Watson, A. (2016). Coping self-efficacy and academic stress among hispanic firstyear college students: the moderating role of emotional intelligence [Cómo afrontar la autoeficacia y el estrés académico entre los estudiantes universitarios hispanos de primer año: el papel moderador de la inteligencia emocional]. Journal of College Counseling, 19(3), 218-230. http://dx.doi.org/10.1002/jocc.12045

Yataco, J. (2019). Bienestar psicológico y estrés académico en estudiantes de la carrera de ingeniería de sistemas de una universidad privada de Lima Sur (Tesis de licenciatura, Universidad Autónoma del Perú). http://repositorio.autonoma.edu.pe/bitstream/AUTONOMA/707/1/Yataco\%20Apaza\%2c $\% 20$ Jessica\%20Paola.pdf

Wang, J., \& Wang, X. (2020). Structural equation modeling: Applications using Mplus (2nd ed.). Hoboken, NJ: Wiley.

Watkins, M. W. (2018). Exploratory factor analysis: A guide to best practice [Análisis factorial exploratorio: una guía de mejores prácticas]. Journal of Black Psychology, 44(3), 219-246. http://dx.doi.org/10.1177/0095798418771807

Wright, S. L., Firsick, D. M., Kacmarski, J. A., \& Jenkins-Guarnieri, M. A. (2017). Effects of attachment on coping efficacy, career decision self-efficacy, and life satisfaction [Efectos del apego sobre la eficacia de afrontamiento, la autoeficacia en las decisiones profesionales y la satisfacción con la vida]. Journal of Counseling and Development, 95(4), 445-456. https://doi.org/10.1002/jcad.12159 